\title{
SIGNIFICADO DE MATERNIDAD EN USUARIAS FEMINISTAS DE INSTAGRAM EN LIMA METROPOLITANA
}

The Meaning of Motherhood in Feminist Instagram Users in Metropolitan Lima

Lucero Brenda Velarde Russo ${ }^{1}$

Correo electrónico: u201312055@upc.edu.pe

1. Universidad Peruana de Ciencias Aplicadas. Facultad de Psicología. Programa Académico de Psicología. (Lima, Perú)

Recibido: 19/10/2020 Aceptado: 19/05/2021

(c) (i) ()ㅜ 


\section{RESUMEN}

INTRODUCCIÓN. En los últimos años el feminismo ha ocupado un espacio mayor dentro de la sociedad peruana. Por lo que se ha vuelto frecuente escuchar debates y opiniones diversas por parte de la población acerca de temas relacionados con este movimiento, siendo el «derecho a decidir ser madre o no» uno de los temas principales y tornando, de este modo, la maternidad en tema de discusión. OBJETIVO. Profundizar en el relato de las participantes para describir y conocer el significado de la maternidad en un grupo específico de mujeres, en este caso, feministas de tercera ola. METODOLOGía. Estudio cualitativo fenomenológico. La técnica utilizada para la recolección de la información fue la entrevista individual semiestructurada. Se llevó a cabo en Lima, Perú. La muestra estuvo conformada por 7 participantes. RESULTADOS Y DISCUSIÓN. Se identificaron tres categorías tras el análisis: (a) Sentidos de maternidad: concepción y experiencia frente a la maternidad; (b) Ser mujer: perspectiva propia y perspectiva de la sociedad; (c) Corporalidad: feminidad y experiencia emocional desde el cuerpo. CONCLUSIONES. Las participantes concuerdan que el cuidado y cariño brindado al infante es lo que define el rol de madre, es decir, este rol deja de ser asignado únicamente al género femenino, dejando en el pasado la ecuación mujer igual madre, de este modo se amplían los horizontes del fenómeno y con ello se comprende la complejidad del mismo.

Palabras clave: maternidad; feminismo; género; estereotipos; roles de género.

\section{ABSTRACT}

INTRODUCTION. In recent years, feminism has occupied a larger space within Peruvian society, and it has become common to hear debates and diverse opinions from the population on issues related to this movement. The right to decide whether or not to be a mother is one of the main issues, making motherhood a topic of discussion. OBJECTIVE. To delve into the participants' accounts to describe and learn about the meaning of motherhood in a specific group of women, in this case, third-wave feminists. METHODOLOGY. A qualitative phenomenological study. The technique used for data collection was the semi-structured individual interview. It was carried out in Lima, Peru. The sample consisted of 7 participants. RESULTS AND DISCUSSION. Three categories were identified after the analysis: (a) Senses of motherhood: conception and experience facing motherhood, (b) Being a woman: own perspective and society's perspective, (c) Corporeality: femininity and emotional experience from the body. CONCLUSIONS. The participants agree that the care and affection given to the infant are what defines the role of mother, that is, this role is no longer assigned solely to the female gender, leaving in the past the equation woman equals mother, thus broadening the horizons of the phenomenon and thus understanding its complexity.

Keywords: Motherhood; Feminism; Gender; Stereotypes; Gender Roles. 


\section{INTRODUCCIÓN}

Según Fuller (2010), desde la modernidad, la identidad femenina ha estado ligada desproporcionadamente a la maternidad. Federici (2010) denomina este fenómeno como «domesticación», la cual propone que el cuerpo humano se transforma en una máquina de trabajo y la mujer toma el rol de «reproductora». Asimismo, Zurian y Herrero (2014) reafirman que la feminidad tradicional gira en torno a la maternidad, pues el proyecto de vida de la mayoría de las mujeres se construye alrededor de su experiencia como madre.

En este sentido, Foucault $(2007 ; 2012)$ explica que la sociedad, gracias al poder, intenta descifrar quiénes somos bajo una lógica universal: el sexo. Según Mondragón (2017), el poder define lo que es normal y lo que no, al reunir un conjunto de saberes reconocidos como legítimos, asignando comportamientos específicos para «varones»y «damas», que resultan en los roles de género. Asimismo, Velázquez (2015) explicó que, a lo largo de la historia, la mujer ha sido situada en una categoría inferior a la del hombre, lo que reforzó su papel secundario en la sociedad.

El feminismo busca proteger los derechos de las mujeres, teniendo como objetivo acabar con el sexismo, la explotación sexista y la opresión del patriarcado (Archenti y Tula, 2019; Hooks, 2017). Con el paso del tiempo se han creado variantes, siendo una de ellas el feminismo de tercera ola que surgió en los años noventa (Snyder, 2008). Esta corriente abraza la multiplicidad de la propia identidad y acepta las contradicciones en la vida misma, rechazando los imperativos del patriarcado y reconciliándose con el rol tradicional femenino desde el propio deseo. De esta forma, la persistencia de lo femenino ya no es un obstáculo para el desarrollo de la autonomía, sino un beneficio (Daros, 2014; Snyder, 2008).

Muñiz y Ramos (2019) refieren que la maternidad es una construcción social que parte de tres aspectos: el biológico, que comprende el embarazo, el parto y la lactancia; el sociocultural, que comprende los ámbitos sociales, históricos e ideológicos, y estructura el concepto de la maternidad; y el subjetivo, que se entiende como la interpretación y la reconstrucción de los significados y conocimientos dentro del contexto social e histórico de la mujer. Es decir, para las feministas de la tercera ola, la maternidad se resignifica a través del deseo de ser madre y no por la opresión social (González, Royo y Silvestre, 2020). 
En la actualidad, las redes sociales son utilizadas para debatir y dar a conocer opiniones públicas. El feminismo virtual (ciberfeminismo) surge en los años noventa y persigue objetivos políticos y estéticos, sirviendo como una red de apoyo y desmintiendo mitos sociales (Branco de Castro, 2015; Lemos, 2009).

En el Perú del año 2016, gracias a la convocatoria por medio de redes sociales, un millón de personas decidieron protestar en contra de la violencia de género en la marcha «Ni una menos» (Caballero, 2018). Es así como Obregon do Nacemento y Klein (2018) plantean que Instagram sirve como una plataforma para que las mujeres feministas expresen sus posiciones ideológicas mediante frases e ilustraciones digitales provocativas.

A diferencia de las mujeres no feministas, estas van contracorriente del status quo; parte de la teoría feminista desmitifica la naturalización e idealización de la maternidad colocando la experiencia personal como centro del fenómeno, lo cual genera tensiones entre lo que se desea y lo que la sociedad exige (Fernández, 2014).

Partiendo de lo mencionado, esta investigación tiene como objetivo describir el significado de maternidad de usuarias feministas de Instagram que comparten contenido relacionado con el empoderamiento femenino, la sexualidad y la libertad del disfrute de esta misma, en Lima, Perú. Así mismo busca responder la pregunta: ¿cómo se manifiesta el significado de la maternidad en las usuarias feministas de Instagram de Lima?

La presente investigación posee relevancia social y busca entender qué sucede dentro de la comunidad peruana feminista perteneciente a la tercera ola, puesto que, como lo explica Turbet (2018), todas las sociedades son patriarcales y, a partir de esto, los movimientos feministas contribuyen a la toma de conciencia y al cuestionamiento de experiencias que se dan por sentadas en la sociedad.

\section{MÉTODO Y MATERIAL}

La presente investigación es cualitativa con diseño fenomenológico; busca analizar las experiencias de las participantes frente un fenómeno particular: la maternidad. Asimismo, Creswell (2018) plantea que la fenomenología permite comprender la experiencia vivida por las personas frente a un fenómeno. 
La investigación tuvo como población usuarias feministas pertenecientes a la tercera ola de Instagram, que actualmente residen en el departamento de Lima-Perú. Asimismo, las participantes pertenecen al nivel socioeconómico A y B, cuentan con viviendas de material noble, seguro de salud particular, estudios superiores, transporte particular, entre otras comodidades (Asociación Peruana de Empresas en Investigación de Mercados, 2016)

La muestra estuvo conformada por siete participantes, entre 24 y 37 años, tres de ellas son madres y cuatro aún continúan evaluando la posibilidad de ejercer la maternidad. Para la elección de las participantes se utilizó el muestreo de bola de nieve, cada participante propone a otros posibles sujetos, y así consecutivamente hasta conseguir una muestra suficiente (Parra y Vázquez, 2017). Al inicio la muestra estuvo conformada por 10 participantes; sin embargo, durante el análisis se procedió a eliminar 3 al no cumplir con el objetivo de investigación.

Por último, la saturación se alcanzó cuando la información se empezó a volver repetitiva (Rodríguez, Garcia y Gil, 1996). A continuación se presenta la Tabla 1 con las principales características sociodemográficas de las entrevistadas. Cabe recalcar que se utilizaron nombres ficticios para proteger la identidad de las participantes.

Tabla 1. Principales características sociodemográficas de las participantes

\begin{tabular}{|l|c|c|c|c|c|}
\hline Nombre & Edad & Estado Civil & $\mathbf{N}^{\mathbf{0}}$ de Hijos & \multicolumn{1}{|c|}{ Distrito } & Ocupación \\
\hline Alessa & 37 & Divorciada & 2 & La Molina & Docente y Free Lance \\
\hline Ange & 28 & Soltera & 0 & San Isidro & Abogada \\
\hline Ani & 24 & Soltera & 1 & Pachacamac & Artista plástica \\
\hline Carmela & 28 & Conviviente & 1 & Miraflores & Fotógrafa \\
\hline Lucía & 25 & Soltera & 0 & Miraflores & Actriz \\
\hline Maca & 26 & Soltera & 0 & Miraflores & Fotógrafa \\
\hline Paola & 26 & Soltera & 0 & Miraflores & Fotógrafa \\
\hline
\end{tabular}

Se utilizó la técnica de entrevista semiestructurada, la cual permite obtener información específica a través de preguntas abiertas y cerradas, lo que permite repreguntar y ser flexible (Martínez, Mañas y Pons, 2016). Para ello se construyó un guion de entrevista que contiene los conceptos teóricos principales del fenómeno de la maternidad, que son el género, la biología, la socioculturalidad y la subjetividad. Este 
instrumento fue supervisado y corregido por 3 especialistas en el tema y posteriormente aplicada. La investigación se desarrolló durante cuatro ciclos académicos. Esta fue monitoreada por especialistas durante el proceso.

Las entrevistas fueron realizadas luego de la aceptación voluntaria de las participantes. Al inicio de la entrevista se entregó un consentimiento informado, documento a través del cual la participante autoriza y acepta voluntariamente ser parte de la investigación. Asimismo, se comunicó que la entrevista sería grabada para, posteriormente realizar una transcripción y analizar la información, y que se mantendría la confidencialidad mediante el uso de un seudónimo. Noreña, Alcaraz, Rojas, y Rebolledo (2012) señalan que estos criterios brindan seguridad y protección a las participantes como informantes para la investigación.

Se utilizaron los criterios de calidad, propuestos por Calderón (2002) y Noreña, et al. (2012). Con respecto a la adecuación epistemológica esta permite obtener información de la subjetividad de las participantes sobre el fenómeno estudiado. Del mismo modo, se comprobó la validez y la fiabilidad de la guía de preguntas mediante una revisión por parte de expertos, y se respetó lo dicho por las participantes a través de la trascripción literal de la entrevista. Esta validez permite al investigador analizar e interpretar la realidad del sujeto desde los antecedentes teóricos y la experiencia del participante. Por último, el criterio de relevancia, en la presente investigación busca indagar acerca del significado de la maternidad, ya que es una situación que incluso hoy en día se impone a la mujer, generando conflictos entre los ideales personales y los esperados por la sociedad, sobre todo en un grupo de mujeres feministas.

Se utilizó el análisis temático. Este método permite identificar, organizar por categorías y subcategorías, y analizar la información obtenida en las entrevistas, mediante las cuales se puede inferir en los resultados para una mayor comprensión del fenómeno de estudio (Mieles, Tonon y Alvarado, 2012).

\section{RESULTADOS Y DISCUSIÓN}

En la presente sección, se expondrán los hallazgos desde lo más general a lo más específico. A partir del proceso de análisis temático realizado, se establecieron tres categorías del significado de maternidad: 1) sentidos de maternidad; 2) ser mujer y 3) corporalidad, como se observa en la Figura 1. 
Figura 1. Categorías del Significado de Maternidad

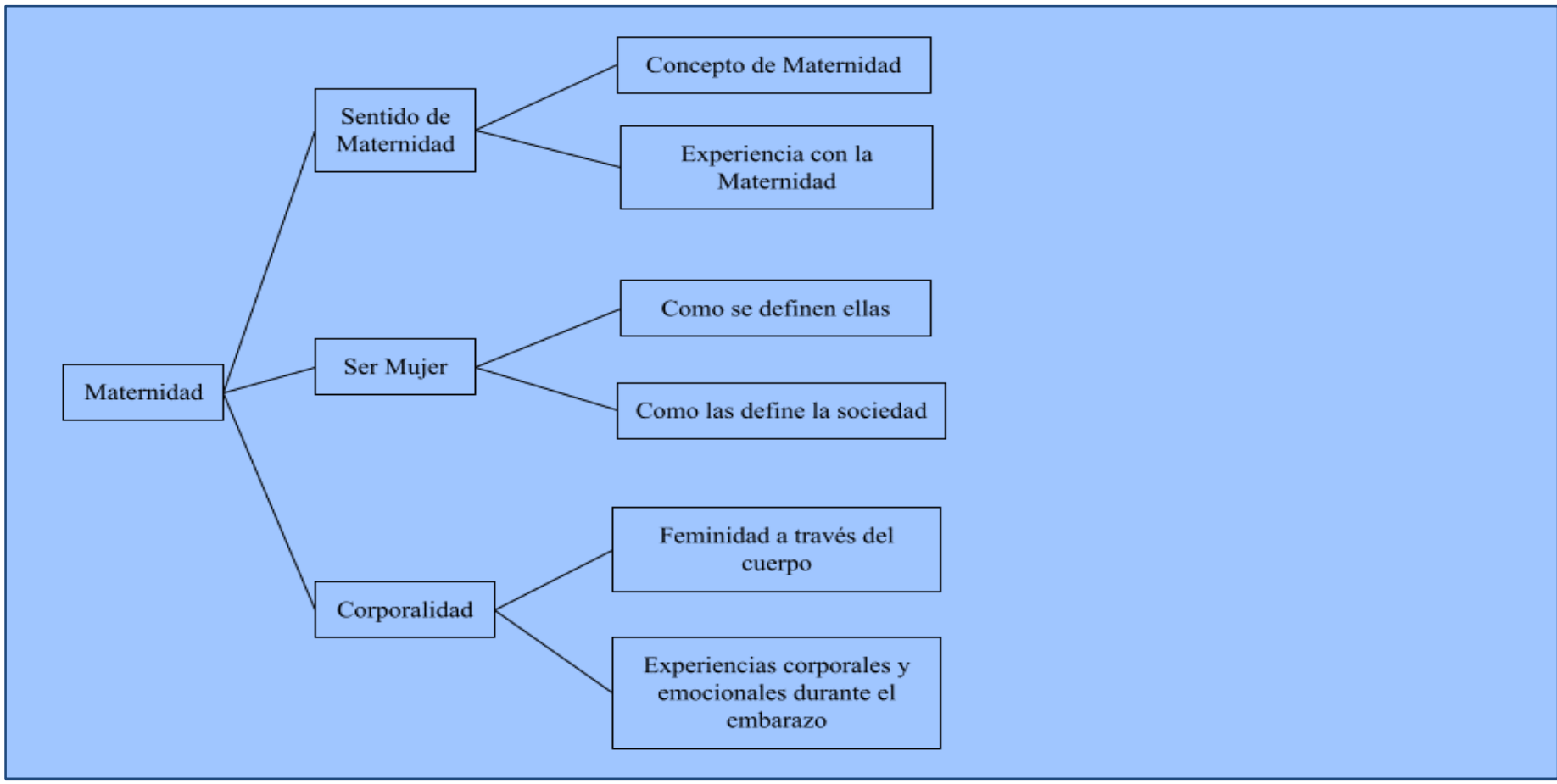

\section{Sentidos de maternidad}

Sentidos de maternidad se encuentra dividida en dos subcategorías. La subcategoría concepto de maternidad muestra que las mujeres que comparten un concepto similar consideran que lo que te hace ser madre es cuidar y proteger al infante.

Lucia (25): [el ser madre es] el querer genuino de cuidar a alguien [...], estar allí para esa persona, aconsejar, en otros momento hacerle darse cuenta de lo está haciendo mal, me parece que no es necesario que tenga que pasar por un proceso biológico.

Este fragmento va en contra de creencias históricas, donde la mujer era la encargada exclusiva de las funciones maternales (Abajo-Llama, et al. 2016). Desde esta perspectiva, se puede comprender que el ser madre va más allá del género. Esta nueva forma de ver a la maternidad permite a la mujer liberarse, dejar los deberes y ejercer sus derechos (Rodríguez, 2020).

Adicionalmente, las entrevistadas expresaron que el ser madre no necesariamente está ligado con el concebir; dar a luz no otorga el título de madre. Roigé (2002) menciona que esto sucede desde el siglo XXI, ya que los conceptos de familia y tipos de maternidad han variado; las parejas tienen hijos sin estar casados, se separan y los niños crecen en hogares 
distintos. La mujer coloca en primer lugar sus metas personales antes de pensar en el matrimonio o concebir.

Asimismo, la posibilidad de decidir ser madre es fundamental para las entrevistadas; en este sentido, hay que tener en cuenta que en el país se realizan al año más de 300000 mil abortos clandestinos (Taype y Merino, 2016). Con ello se puede concluir que en Perú muchas mujeres no desean ejercer la maternidad, pero son obligadas a ello, ya que la ley no permite a la mujer interrumpir el embarazo en caso lo desee. Sutton y Borland (2017) explican que el aborto es un derecho, catalogado como una deuda de la democracia hacia la mujer, dado que por mucho tiempo esta fue reprimida de sus propios derechos.

Alessa (37): Madre es la que cría [...] dar a luz no significa nada y por esto estoy [...] a favor del aborto [...] hay muchas mujeres que son obligada a parir y el amor y la maternidad no se les desarrolla.

Las participantes ven a la maternidad como algo complejo que involucra protección y cuidado y no corresponde solamente a la mujer. Romero, Tapia y Meza (2020), junto con Rodríguez (2001), explican que la «mujer madre» actual redefine su identidad en oposición directa al patriarcado, decidiendo ser madre a voluntad. Asimismo, Romero, et al. (2020) manifiestan que las ideas y maneras de ejercer la maternidad actual son diferentes a la concepción tradicional.

Maca (26): [...] cualquiera puede ser madre, [...] esto no solo se relaciona con ser mujer. Puede existir un padre que sea padre y madre a la vez. [...] para mí todo depende de cuanta atención tu hijo merezca [...].

La idea que expresa Maca (26) es distinta a lo convencional, debido a que el Perú se ciñe por normas conservadoras. Tello (2019) explica como diversos movimientos ligados a la religión y organizaciones profamilia y antiaborto, son una gran traba al momento de querer promover leyes que protejan y velen por los derechos de las minorías, como la comunidad LGTBIQ o grupos feministas. En relación con el concepto «familia», Barceló (2016) explica que actualmente existe una «familia posfamiliar», la cual es cambiante y múltiple, que no se rige por ideas tradicionales. En Maca (26) se puede apreciar este concepto, afirmando que el género es independiente del rol de madre y la atención que se le brinda al infante. No obstante, la población peruana lo considera antinatural, debido a que cualquier relación que no esté conformada por una pareja heterosexual va en contra de la naturaleza de la familia tradicional (Tello, 2019). 
Paola (26): [...] bueno es una elección y nada más, más que el «filin» opino que es una decisión que las mujeres pueden decidir si desean ser mamás.

Para algunas participantes, la elección de ser madre es una decisión personal. Romero, et al. (2020) explican que, en Occidente, antes de la aparición de los estudios feministas, se consideraba a la maternidad como una cuestión inherente a las mujeres, cuyo origen incluso se asociaba a mandatos divinos. En esta participante se puede apreciar la influencia del feminismo en su pensar, como es que sus creencias personales influyen y crean una nueva concepción de maternidad.

Carmela (28): [...] se les decía a las mujeres que su rol en la vida debía ser madres [...], que si no tienen hijos no se realizaban como personas. [...] estoy en contra de esa idea me aparece absurda, ridícula y machista. Pero al mismo tiempo, yo no hubiera querido no tenerlo [habla de su hijo]

La subcategoría experiencia con la maternidad se basa en los conocimientos adquiridos por las participantes a lo largo de sus vidas en relación con la maternidad. En el caso de Carmela (28), menciona el rechazo a la idea de mujer igual madre y enfatiza la cadencia de obligación a ejercer la maternidad. Luna y Mejía (2017) exponen que en la actualidad en Latinoamérica se ha disminuido la tasa de natalidad debido al alto uso de métodos anticonceptivos, puesto que las mujeres eligen una profesión o no desean tener un hijo. No obstante, la participante expresa que ella sí deseaba la maternidad. Abajo, et al. (2016) explican que en la actualidad la maternidad es un signo de revolución para las feministas, debido a que el deseo de un hijo se convierte en la reivindicación de un derecho. Es decir, para Carmela (28) el tener un hijo ha sido una manera de exigir ante la sociedad su derecho de ser madre, sin conectarlo a la obligación social por su género.

También se halló que la experiencia o contacto con la maternidad ha sido compleja para las participantes. Comentan que es un trabajo arduo y duro, donde la mujer pasa a dar todo de sí misma al bebé. Horowitz (2001) y Romero et al. (2020) comparten los pensamientos de Betty Friedan (1963), quien hace énfasis sobre el rol que tiene la mujer dentro de casa, alegando que existe un «malestar que no tiene nombre» cuando se cumple este papel. Daros (2014) considera que en la actualidad algunas mujeres rechazan el modelo masculino de solo trabajar y dejar de lado los sentimientos y la comunicación, reconciliándose con el rol tradicional femenino, lo cual no es un obstáculo hacia la autonomía, sino un enriquecimiento de una misma. 
Ani (24): Un recién nacido te absorbe [...] tú eres su mundo [...] tu cuerpo cambia más cuando estás dando de lactar, te das cuenta que ya no puedes hacer las cosas como antes.

Desde la perspectiva de Ani (24), siente que su hija la absorbe, probablemente ello derive de la concepción tradicional del ser madre. Beg (2020) explica que aún existe una imposición de ideas, reglas y regulaciones por parte de la sociedad que la mujer tiene que cumplir, puesto que la mujer debe conservar una imagen perfecta como madre sin importar sus emociones y deseos. Pese a ser feminista y vivir bajo este constructo social, la participante aún se siente atrapada en el estereotipo de madre, ya que de no hacerlo, no estaría cumpliendo con las normas establecidas.

\section{Ser mujer}

Con respecto a la categoría ser mujer, esta parte de la propia perspectiva que tienen las participantes del ser mujer y la opinión que tienen de cómo la sociedad considera a la mujer. Las subcategorías son cómo se definen ellas y cómo las define la sociedad.

Se encontraron diferencias entre las participantes madres y las que no lo son. Las participantes madres describieron «qué es ser mujer» desde su propia maternidad, sin separar su rol de mujer y madre. Esto puede darse debido a como su rol de madre ha ocupado la mayor parte de su identidad. Agudelo, Bedoya y Osorio (2016) afirman que las madres con niños pequeños priorizan las necesidades y demandas de sus hijos, lo cual debería cambiar mientras crece. Matláry (2002), como se citó en Abajo et al. (2016) relaciona a la maternidad con la toma de conciencia personal de quiénes somos, es decir, al experimentar la maternidad la persona se sumerge en una reidentificación de uno mismo, por lo que es complejo desligar su rol de madre al de mujer.

Carmela (28): [...] yo siempre decía que no quería tener hijos en el fondo era mentira $[\ldots]$ estoy súper feliz con su existencia, por eso te digo que eso me ha enseñado una dimensión totalmente nueva de mí que antes no conocía.

Por otro lado las participantes que no son madres brindaron una respuesta concreta en relación con lo que para ellas es ser mujer.

Paola (26): [...] para mí ha sido difícil sentirme cien por ciento mujer, porque desde chiquita me han metido el estereotipo de mujer y desde niña era totalmente distinta, han pasado años de años de años [...] he luchado, de allí 
ha sido aceptación. [...] para ser mujer [...] solo lo debes sentir y si te identificas así bravaso no creo que implique ropa estilos, tipo de voz aspecto nada.

Maca (26): [...] para mí ser mujer es una mezcla de intuición con inteligencia y también significa tener un rol en esta sociedad que me obliga a más.

Estas frases expresan la presión que la sociedad ejerce por el hecho de ser mujeres. Por un lado Paola (26) narra que se ha sentido vulnerada por ser mujer dentro de la sociedad. Adicionalmente, Maca (25) suma la necesidad de hacerse valer, debido al papel secundario que tiene la mujer socialmente. El significado que las participantes le dan al ser mujer ha sido construido en base a conceptos que no son compartidos socialmente. Federici (2004) y Mannareli (2018) explican que, desde la época de la caza de brujas, la mujer ha ido perdiendo poder en la sociedad a causa de la violencia y la domesticación. Para las autoras, las participantes serian consideradas brujas en el siglo XVI. En la actualidad no se les llama de esta forma pero se les considera menos femeninas por tratar de hacerse valer, ser inteligentes y libres sexualmente.

Por otro lado, la subcategoría cómo las define la sociedad resultó en un descubrimiento de estereotipos compartidos. Todas mencionaron que la madre peruana es abnegada, siempre tiene que estar de buen humor, es la encargada de los quehaceres del hogar y cuidado familiar; de igual forma, expresaron que estas definiciones provienen del fuerte machismo que existe en el país. Aún en pleno siglo XXI dentro de la sociedad peruana, la mejor salida que tiene la mujer para crearse una identidad propia es el convertirse en madre, puesto que aún el ser madre brinda el estatus de adulto y reconocimiento social (Fuller, 2010). Igualmente, Fuller (2001) relaciona este problema con la gran diferencia de poder que existe entre el hombre y la mujer, dificultando acceso a las mismas oportunidades, sobre todo si se proviene de sectores sociales o grupos étnicos minoritarios.

Alessa (37): [...] La sociedad impone roles en las mujeres, sobre todo de cuidadora y de madre. Si no te llegues a casar de grande vas a tener que cuidar [...], esos son los principales roles que nos dan. [Acerca de la madre en Perú] Es sacrificada, [...] el tema del sacrificio y la culpabilidad están bien amarradas [con la maternidad], Existe esa imagen cero mujer, te vuelves solo mamá, te dedicas solo a los hijos, en general a ser un ama de casa completa y 
si trabajas entonces vas hacer excelente tu trabajo y excelente ama de casa también, y vas a ser como la súper mujer que te encargas de todo [...]. Te ponen toda una montaña de responsabilidades.

\section{Corporalidad}

Se encontró que las participantes tienen una relación significativa con su cuerpo. En la subcategoría feminidad a través del cuerpo se puede apreciar que el cuerpo brinda un sentido de identidad.

Ani (24): a través de la foto podía encontrar un ángulo de una tercera persona de un aspecto mío que quizás no veía. [...] mostrar piel [...] empoderar el cuerpo. [...] para mí las fotos son una manera de mostrarme.

En el caso de Aní (24), retratarse la ayuda a entender quién es ella y crear un insight propio. Explica que para ello fue necesario tener la valoración de otro sobre sí misma, lo cual hace referencia al poder que la sociedad tiene sobre la mujer. El cuerpo es el escenario en el cual la persona crea la diferencia entre el sujeto y el mundo, es decir, la imagen del cuerpo se mediatiza por el reflejo en forma de espejo frente a los demás o por el modo en que los otros te miran (Romero y García, 2018).

Lucía (25): [...] yo en Instagram he publicado fotos calatas, [...] esa es la manera de lidiar con mis inseguridades con mi cuerpo, con lo que a mí me atrae estéticamente.

De igual forma, Lucia (25) menciona que es el cuerpo el que permite lidiar y entenderse con una misma. Fuller (2001) menciona que algunas mujeres desde el siglo XX en adelante ven el cuerpo y la sexualidad como algo normal, se rechaza la doble moral sexual y se considera injusta, afirmando que es necesario superar la inhibición sexual. De esta manera, se puede alcanzar salud psíquica, ya que el cuerpo deja de ser prisionero de las expectativas sociales y pasa a pertenecer a una misma.

La última subcategoría experiencias corporales y emocionales durante el embarazo explica cómo es que desde el cuerpo de uno o de otros se pueden producir diferentes emociones, tanto positivas como negativas durante la experiencia frente al embarazo. 
Carmela (28): [...] es bien importante ir preparándote no solo como crece él bebé y tu barriga, sino que cada vez vas desarrollando un vínculo en tu mente y en tu corazón [...]. El amor por tu hijo es más fuerte que cualquier cosa no solo por tu mascota, si no por tus propios padres o por tu pareja, que son los amores más fuertes.

La participante refiere que el amor que tiene por su hijo es mayor a cualquier otro. Esto podría hacer pensar que se refiere al amor maternal; sin embargo, para García (2019) este amor maternal no existe, es tan solo una muestra más de una creación histórico-social que varía según las épocas y costumbres. Esto muestra como la cultura ha ido moldeando su manera de sentir frente a su hijo. Adicionalmente, Carmela (28) menciona que durante el embarazo afloran nuevas emociones y sentimientos que se dan por los cambios que el cuerpo produce. Estos cambios crean un nuevo vínculo entre la mujer y el niño, y no siempre van a ser positivos. Abajo et al. (2016) manifiestan que durante el embarazo la mujer crea expectativas e imagina cómo deberían suceder los hechos durante el parto como durante la crianza. No obstante, la realidad es diferente a lo esperado, lo que genera sentimientos de culpa y frustración, por no ser lo suficiente buena madre. García (2019) refiere que la sociedad influenciara en la mirada que tiene la madre de sí misma, es decir, la mujer será o no será buena madre en función de lo que la sociedad desprecie o valorice de la maternidad.

Paula (26): [...] ha habido chicas que he conocido que salieron embarazadas y ha sido un cambio tan brusco que ha sido traumático entonces creo que es muy importante tomar en cuenta lo se te viene al cuerpo.

Paula (26) menciona que las mujeres, al no estar preparadas para los cambios del embarazo, se angustian, convirtiéndolo en un proceso traumático, debido a la falta de preparación. Esta situación aleja a la mujer del bebé y de sí misma. Palacios (2009) refiere que el cuerpo es el escenario de las vivencias del ser humano, por el cual pasa el tiempo y muestra los estragos de este mismo. Durante el embarazo estos cambios son continuos hasta el nacimiento del bebé. Arcos (2017) explica estos malestares por el cuerpo desde la biopolítica de lo materno, donde la mujer es protagonista de roles y una estética especifica. Estas expectativas sociales se cruzan con las propias durante el embarazo, creando nuevas dudas y reclamos, provocando así sentimientos de malestar e incomodidad. 


\section{CONCLUSIONES}

Se puede concluir que el significado de maternidad no se puede definir sin tomar en cuenta lo biológico, lo sociocultural y lo subjetivo. No obstante, se pudo apreciar que la maternidad desde el punto de vista de las participantes difiere de la visión tradicional, puesto que, al ser mujeres feministas de tercera ola, los conocimientos e información que manejan frente al fenómeno varían substancialmente frente al resto y según la experiencia de cada persona.

El sentido que le dan a la maternidad parte de sus propias creencias. Consideran que para ser catalogada como madre solo basta brindar amor, cuidado y protección al infante, deslingando las tareas a un género especifico. Adicionalmente, la opción de decidir ser madre es fundamental para las entrevistadas; gestar desde el deseo reivindica su derecho como mujer, ya que proviene de una misma y no de lo que la sociedad espera de ellas.

Por otro lado, las participantes que son madres mencionan que tanto su rol de mujer como madre están relacionados, ya que cuando se comienza a ejercer la maternidad las demandas de este rol se priorizan. Por el contrario, las participantes que no son madres definieron el ser mujer sin contar la maternidad, afirmando que constantemente deben hacerse valer ante la sociedad para tener un lugar en esta. No obstante, todas las participantes concordaron que la visión que tiene la sociedad frente a la madre está llena de estereotipos, según los cuales la mujer debe tener como rol principal ser la encargada de los cuidados de la familia, lo cual obstaculiza su desarrollo individual como persona autónoma.

Asimismo, se encontró que las participantes tienen una relación significativa con su cuerpo, ya que refieren que durante el proceso de embarazo y maternidad suceden cambios tanto físicos como psíquicos, debido a que este determinará el vínculo con el infante y el rol de madre.

Finalmente, en cuanto las limitaciones del estudio, la primera fue encontrar la muestra. Pesé a que existe un número sustancial de mujeres feministas en Lima, no todas cumplían con los criterios de inclusión. Por otro lado, la recolección de información se realizó durante un semestre académico, lo cual acelero el proceso de entrevista, ya que había una fecha de entrega. 


\section{BIBLIOGRAFÍA}

ABAJO-Llama, Susana, et al. Ser madre hoy: abordaje multidisciplinar de la maternidad desde una perspectiva de género. En: MUSAS. Revista de Investigación en Mujer, Salud y Sociedad. 2016, vol. 1, núm. 2.

Agudelo Londoño, Jesica; Bedoya García, Johana; Osorio TAMAYo, Dora. Ser mujer: entre la maternidad y la identidad. En: Poiésis. 2016, núm. 31.

Amoros, Celia; De Miguel, Ana. Teoría Feminista: de la ilustración a la globalización. España: Minerva Ediciones, 2005.

ARCHENTI, Nélida; Tula, María. Teoría y política en clave de género. En: Colección. 2019, vol. 30, núm. 1.

Arcos Herrera, Carol. Feminismos latinoamericanos: deseo, cuerpo y biopolítica de lo materno. En: Debate Feminista. 2018, vol. 55.

Asociación Peruana de Empresas de Investigación de Mercados (APEIM). Niveles Socioeconómicos. Lima: APEIM, 2016, [consulta:15 de abril 2020]. Disponible en: $<$ http://apeim.com.pe/wp-content/uploads/2019/11/APEIM-NSE-2016.pdf> .

BARCEló Tous, María. Un camino hacia la maternidad pospatriarcal. En: Revista de Antropología Iberoamericana. 2016, vol. 11, núm. 1.

BEG, Sadiqa. Embedded Misogyny: Objectification of Women in Indian Society. En: Our Heritage. 2020, vol. 68.

Branco de Castro Ferreira, Carolina. Feminisms on the web: lines and forms of action in contemporary feminist debate. En: Cad. Pagu. 2015, núm. 44, pp. 199-228.

CABALlERO, Gerardo. Redes sociales y feminismos en la acción colectiva: el caso de «Ni Una Menos» en el Perú. [Tesis de máster]. Pontificia Universidad Católica del Perú, 2018.

CALDERÓn, Carlos. Criterios de calidad en la investigación cualitativa en salud (ICS): apuntes para un debate necesarios. En: Revista Española de Salud Pública. 2002, vol. 76, núm. 5.

CREswell, John. Qualitative Inquiry and Research Design: Choosing Among Five Approaches. Estados Unidos: SAGE publications, 2007.

Creswell, John; Creswell, David. Research design: qualitative, quantitative, and mixed methods approaches. Los Ángeles: SAGE, 2018.

Creswell, John; Poth, Cheryl. Qualitative Inquiry and Research Design: Choosing Among Five Approaches. Estados Unidos: SAGE publications, 2018.

DAROS, William. La mujer posmoderna y el machismo. En: Franciscanum. Revista de las ciencias del espíritu. 2014, vol. LVI, núm. 162.

FeDERICI, Silvia. Caliban y la bruja. Mujeres, cuerpo y acumulación originaria [online]. Madrid, España: Traficantes de sueños, 2010. [Consulta: 7 de marzo de 2021]. Disponible en: https://bit.ly/3wSWJXJ

FERNÁNDEZ, Irati. Feminismo y Matenidad: ¿Una relación incómoda? País Vasco: Emakunde/Instituto Vasco de la Mujer, 2014. 
Flecha, Ainhoa. Las hijas de las feministas. El feminismo en el siglo XXI ¿declive o democratización? En: RASE. 2016, vol. 3, núm. 3.

FoucAult, Michel. Historia de la sexualidad. 1. La voluntad del saber. México: Siglo XXI editores, 2007.

Foucault, Michel. The mesh of power. En: Viewpoint Magazine. 2012, núm. 2.

FULLER, Norma. Identidad femenina y maternidad: una relación incómoda. En: DEMUS. 2010.

Disponible en:

http://red.pucp.edu.pe/wp-content/uploads/biblioteca/081008.pdf

FULLER, Norma. Maternidad e identidad femenina: relato de sus desencuentros. En: Donas

Burak, Solum (ed.). Adolescencia y Juventud en América Latina. Cartago: Libro Universitario Regional, 2001, pp. 225-242.

GonZales, Lía; Royo, Raquel; Silvestre, María. Voces de mujeres jóvenes feministas ante la maternidad: deconstruyendo el imaginario social. En: Investigaciones Feministas. 2020, vol. 11, núm. 1, pp. 31-41.

Hooks, Bell. El feminismo es para todo el mundo. En: Sudamérica. 2017, núm. 10, pp. 146150.

Horowitz, Daniel. Betty Friedan and the Making of the Feminine Mystique: The American Left, the Cold War, and Modern Feminism. Estados Unidos: University of Massachusetts Press, 2001.

Lemos, Marina Gazire. Ciberfeminismo: novos discursos do femenino em redes electrônicas. [Tesis de máster]. Pontifícia Universidade Católica de São Paulo, 2009.

Luna GIRALdO, Leidy; MeJ́a MaCias, Angi. ¿Por qué no quiero ser madre? Un estudio sobre la configuración subjetiva de la no maternidad. [Tesis de licenciatura]. Corporación Universitaria Minuto de Dios, Facultad de Ciencias Humanas y Sociales, Programa de Psicología, 2017.

MARTíN-GARCÍA, Teresa. «¿Existe el amor maternal? Historia del amor maternal. Siglos XVII al XX» de Elisabeth Badinter. En: Encrucijadas. Revista Crítica de Ciencias Sociales. 2019, vol. 18 .

Mondragón Lazo, Viviana. Teoría del poder y violencia de género. En: Pluralidad y Consenso. 2017, vol. 7, núm. 31.

MuÑIz Gallardo, Erika; Ramos Tovar, María. Presión social para ser madre hacia mujeres académicas sin hijos. En: Nóesis. Revista de Ciencias Sociales y Humanidades. 2019, vol. 28, núm. 55.

Noreña, Ana, et al. Aplicabilidad de los criterios de rigor y éticos en la investigación cualitativa. En: Aquichan. 2012, vol. 12, núm. 3.

OBRegOn do NAscimento, Bianca; KLEIN, Eloisa. As estratégias de engajamento de perfis feministas no instagram. En: Anais Do Salão Internacional De Ensino, Pesquisa E Extensão. 2018, vol. 10, núm. 2. 
PARRA, Laura; VÁZQUEZ, María Guadalupe. Muestreo probabilístico y no probabilístico. Los Pueblos: Universidad del Istmo, 2017.

Rodriguez, Alfonsa. La familia posmoderna: distancia y compromiso. En: Redes. Revista de Psicoterapia Relacional e Intervenciones Sociales. 2001, núm. 8.

RodríGuez GómEz, Gregorio; GARCÍA JiMÉNEZ, Eduardo; GIL FlorES, Javier. Metodología de la investigación cualitativa. España: Editorial Aljibe, 1996.

Romero GuzMÁn, María; TAPIA Tovar, Evangelina; Meza MÁrquez, Consuelo. Abanico de maternidades. Un estado del arte desde los aportes del feminismo. En: Debate Feminista. 2019, vol. 59 .

Romero Zuñiga, Patrícia; Garcia Peñafiel, Mauricio. Mujer, madre, hija: discursos e imágenes del cuerpo en la adicción femenina y sus relaciones con la trasmisión de la feminidad. Estudio cualitativo en mujeres chilenas. En: Revista Latinoamericana de Psicopatologia Fundamental. 2018, vol. 21, núm. 3.

SÁNCHEZ BRINGAS, Ángeles, et al. Nuevas maternidades o la deconstrucción de la maternidad en México. En: Debate Feminista. 2004, vol. 30.

SuTTON, Bárbara; BORLAND, Elizabeth. El discurso de los derechos humanos y la militancia por el derecho al aborto en la Argentina. En: Horizontes revolucionarios. Voces y cuerpos en conflicto. 2017.

SNYDER, Claire. What Is Third-Wave Feminism? A New Directions Essay. En: Signs. 2008, vol. 34, núm. 1, pp. 175-196.

Taype-Rondan, Alvaro; Merino-Garcia, Nicolaz. Hospitalizaciones y muertes por aborto clandestino en Perú: ¿qué dicen los números? En: Revista Peruana de Medicina Experimental y Salud Publica. 2016, vol. 33, núm. 4.

Tello Aguinaga, Kevin. La cruzada contra la ideología de género: causas de la politización del conservadurismo evangélico en el Perú contemporáneo. [Tesis de licenciatura]. Pontificia Universidad Católica del Perú, 2019.

TuBert, Silvia. Psicoanálisis, feminismo y posmodernismo. En: Errancia. 2018, vol. 17.

VelázQuez Herrera, Luisa. Redes Sociales y Activismo Feminista. En: UNESCO [online]. [Consulta: 10 de julio 2020]. Disponible en:

http://www.unesco.org/new/fileadmin/MULTIMEDIA/HQ/CI/CI/pdf/panel_1_4 luisa_v elazquez_spanish.pdf

WILDING, Faith. ¿Dónde está el feminismo en el ciberfeminismo? En: Lectora: revista de dones i textualitat. 2004, núm. 10, pp. 141-152.

Zurian Hernández, Francisco; HerRero JiméNEZ, Beatriz. Los estudios de género y la teoría filmica feminista como marco teórico y metodológico para la investigación en Cultura Audiovisual. En: Área Abierta. 2014, vol. 14, núm. 3. 